\title{
Information and Communications Technology Metaphors
}

\author{
Ömür Akdemir ${ }^{1 *}$, Dinçer Biçer ${ }^{1}$, Ramazan Ş. Parmaksız ${ }^{1}$
}

${ }^{1}$ Bulent Ecevit University, Zonguldak, TURKEY

*Corresponding Author: omurakdemir@gmail.com

Citation: Akdemir, Ö., Biçer, D., \& Parmaksız, R. Ş. (2020). Information and Communications Technology Metaphors. Mediterranean Journal of Social \& Behavioral Research, 4(1), 11-18. https://doi.org/10.30935/mjosbr/9596

\begin{abstract}
Determination of the perceptions of the prospective teachers for the ICT terms have a remarkable potential to provide input for technology integration plans and ICT trainings. Within this context, the purpose of this study is to discover the metaphors constructed by prospective teachers for the ICT terms. Data were gathered from 180 prospective teachers through survey. 977 valid metaphors constructed by the participants were grouped into conceptual categories for the six ICT terms. The most common conceptual categories are "developing and changing" for the technology, "making life easy" for computers and search engines, "limitless and endless" for the Internet, "means of communication" for social networks, and "addictive items" for video games.
\end{abstract}

Keywords: metaphors, ICT, teacher education, prospective teachers

Received: 28 Oct. $2019 \bullet$ Revised: 01 Feb. $2020 \bullet$ Accepted: 16 Mar. 2020

\section{INTRODUCTION}

The use of technology has recently become more common than ever before. Along with the use of technological devices, this has also brought about the frequent use of words related with technology in our daily lives and in education. Developments in Information and Communications Technology have opened a great avenue for educators to improve teaching and learning. Technology is currently perceived in education as a mean of enhancing teaching and learning. In order to embed technology efficiently in educational processes, it is necessary for teacher educators to know how technology and terms related to the technology are perceived by prospective teachers. When teacher candidates have sufficient instruction during their education they have positive ideas for instruction using technology and they believe that it is effective (Morrison and Jeffs, 2005).

A significant quality of technology is that it eliminates limits in education, transferring learning processes outside the class enabling learning during the entire day and life. This also supports life-long learning which has become quite popular recently due to the fact that learning must not be limited within education offered at school, it should continue during entire life because new knowledge is generated very rapidly. In order to make use of technology in teaching to support limitless learning, and to grant learners with life-long learning skills, it is required for teacher educators to know how students perceive forms of technology such as social networks, search engines and video games. It has been indicated that prospective teachers' behavioral approaches perceptions of the technologies that they are going to make use of in their classrooms have a strong influence on their future perceptions and eagerness to use technology in their teaching environments (Teo and Lee 2010).

Studies investigating metaphors used for the computer and for the technology can be found on the literature (Gecer, 2013; Gurol \& Donmus, 2010; Kobak \& Taskin, 2012; Koc, 2013). However there is no single study investigating the metaphors for the terms used in Information Communications Technology which are frequently used by the stakeholders of the education adventure. Prospective teachers play the key role in the integration and use of the ICT in schools. Determination of the perceptions of the prospective teachers for ICT terms have a remarkable potential to provide input for technology integration plans and ICT trainings. Within this context, the purpose of this study is to discover the metaphors constructed by prospective teachers for the ICT terms. This study aims to seek answers for the following research questions:

1. Which metaphors do prospective teachers use to portray the terms for technology, computers, the internet, search engines, social networks and video games?

2. How frequently are the metaphors used by prospective teachers to portray the terms for technology, computers, the internet, search engines, social networks and video games?

3. How do prospective teachers justify metaphors for the ICT terms that they constructed? 
Table 1. Distribution of the participants according to their gender and programs

Undergraduate Program

\begin{tabular}{|c|c|c|c|c|c|c|c|}
\hline & & \multicolumn{2}{|c|}{ Early Childhood Ed. } & \multicolumn{2}{|c|}{ Special Ed. } & \multicolumn{2}{|c|}{ Total } \\
\hline & & $f$ & $\%$ & $\mathbf{f}$ & $\%$ & $f$ & $\%$ \\
\hline \multirow{2}{*}{ Gender } & Male & 12 & 11,8 & 31 & 39,2 & 43 & 23,8 \\
\hline & Female & 89 & 88,1 & 48 & 60,7 & 137 & 76,1 \\
\hline Total & & 101 & 100 & 79 & 100 & 180 & 100 \\
\hline
\end{tabular}

\section{METHODOLOGY}

\section{Design of the Study}

The qualitative survey (Jansen, 2010), recently emerging type of qualitative research, was used in the study. There were two open-ended questions at the survey. The first open-ended question was targeting to gather the metaphors constructed by the prospective teachers for the six ICT terms. The second open-ended question was asking respondents to justify the metaphors for the ICT terms that they constructed. The content analysis was used to analyze the qualitative data.

\section{Participants}

The participants of the study are 180 freshmen prospective teachers studying at the Education Faculty of a State university located at the Black Sea region. Prospective teachers studying at the Early Child Education and the Special Education departments in 2013-2014 academic year were selected among volunteers. The distribution of the participants according to their departments and genders are provided in Table 1.

\section{Data collection Instrument}

Participants were asked to complete six questions at the survey to form metaphors. Each survey question was constructed asking participants to write the metaphor for the ICT term and the reason for choosing that metaphor.

\section{Data Analysis}

Participants answered a total number of 1080 questions for the six ICT terms. The first stage of the data analysis includes exclusion and coding. Preliminary analysis of the data revealed that some participants did not use metaphors at the answers. Therefore, these answers were eliminated and not used at the analysis. Similarly, some participants did not provide explanation for the metaphors at the survey, so their answers were not considered at the analysis either. After the elimination of the unacceptable answers, the total number of metaphors left for analysis was 977.

In the next stage, the content analysis process was initiated. Metaphors were alphabetically listed and a sample explanatory sentence describing the metaphor best was selected for each metaphor. After that, the metaphors formed by the participants were analyzed in terms of their common features related with the keywords. Conceptual categories were formed for each keyword associating each metaphor with a certain theme.

\section{Results}

Findings for the first research question

The conceptual categories formed after the elimination of irrelevant or lacking data for each keyword are provided in Table 2.

Findings for the second research question

The frequencies and percentages of the conceptual categories formed after the elimination of irrelevant or lacking data for each keyword are provided in Table 3.

Findings for the third research question

The most common means by which prospective teachers justify the metaphors they constructed for the ICT terms are provided in Table 4.

\section{CONCLUSION AND RECOMMENDATIONS}

The purpose of this study is to discover the metaphors constructed by prospective teachers for the ICT terms. Results revealed that the most common conceptual category are developing and changing for the technology, making life easy for the computers and search engines, limitless and endless for the Internet, means of communication for the social networks, and addictive items for the video games. These findings reflect the perceptions of prospective teachers on ICT terms.

It has been emphasized in several studies that technology implementation requires profound changes in the role of teachers and their epistemological views (Koc, 2013). Learning the perception of the prospective teachers for the ICT terms is important to take protective actions to change the undesirable perceptions of the prospective teachers for the ICT which is widely used in schools. In Koç (2013)'s study, it has been emphasized that student teachers' conception of technology is restricted, focusing mostly on artifact and technical dimensions. In order to avoid this restriction, the curriculum must have a broader approach towards technology. Future research should concentrate on investigating the match and mismatches between intended use of the ICT tools and the perception of the prospective teachers. 
Table 2. Categories formed for the keywords

\begin{tabular}{|c|c|c|}
\hline Keywords & Conceptual Categories & Metaphor Names \\
\hline \multirow{13}{*}{ 突 } & Developing and changing & $\begin{array}{l}\text { flower, plant, tree, human, child, river, baby, chameleon, living thing, earth, city, life, body, infinity, day, progress, } \\
\text { horse, life, capitalism, black hole, writing, hairdresser, monkey, seasons, water, numbers, historic identity, outer } \\
\text { space, pyramid, rain }\end{array}$ \\
\hline & Limitless, endless & Earth, sky, outer space, tree, living thing, circle, avalanche, abyss, line in geometry, dreaming, numbers \\
\hline & Making life easy & mother, robot, Hercules, popeye, spiderman, a hero performing magic, scientist, donkey, Earth, girl \\
\hline & Necessities & water, tree, bread, sun, life, light, organs, market, inspector gadget, Zeus \\
\hline & Tools & gear, vacuum cleaner, F5 key, pencil, box, hourglass, mixer, kitchen tools, toy \\
\hline & Both beneficial and harmful & humans, nuclear energy, weapon, dinosaur, sun, sea, life \\
\hline & Rapidly progressing & cheetah, rocket, dream, time, surfing \\
\hline & Small animals & bird, louse, butterfly, virus \\
\hline & Food & walnut, water, fried egg with meat \\
\hline & Harmful items & bomb, sandwich with jam, cat \\
\hline & Hardworking animals & bee \\
\hline & Big animals & lion \\
\hline & Addictive items & Nutella \\
\hline \multirow{14}{*}{ 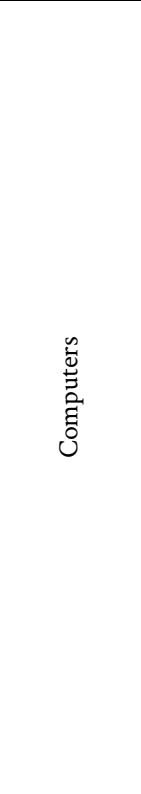 } & Making life easy & $\begin{array}{l}\text { library, intelligence, colt, robot, heartless person, octopus, mother, archive, myself, empty land, house, suitcase, sun, } \\
\text { dough, intelligent person, human, the Jetsons, women, kangaroo, ant, turtle, all-inclusive thing, wise man, kitchen, } \\
\text { teacher, rucksack, superman }\end{array}$ \\
\hline & Organs & brain, veins of brain, veins, heart, nervous system, body \\
\hline & Limitless, endless & $\begin{array}{l}\text { Earth, universe, box, jigsaw puzzle, a lady's bag, living things kingdom, factory, safe, Matruska doll, item, } \\
\text { encyclopedia, vacuum cleaner, road }\end{array}$ \\
\hline & Tools & $\begin{array}{l}\text { box, refrigerator, flight recorder, notebook, cupboard, memory stick, women's memory, cauldron, book, bookshelf, } \\
\text { jewelry box, television }\end{array}$ \\
\hline & Necessities & darling, spiced chips, building base, living thing, washing machine, limbs, house, book, chicken, girl, life, meal \\
\hline & Beneficial items & human, close friend, cow, turtle, encyclopedia, fun box, vacuum cleaner, book \\
\hline & Both beneficial and harmful & corn, human, knife, television, robot \\
\hline & Addictive items & chocolate, honey, virus, cigarette \\
\hline & Big animals & lion, dog, Cyclops \\
\hline & Developing and changing & chameleon, Sultan Mehmet the Conqueror, Metehan \\
\hline & Vehicles & car, truck \\
\hline & Hardworking animals & ant \\
\hline & Means of communication & telephone \\
\hline & Small animals & puppy \\
\hline \multirow{13}{*}{ 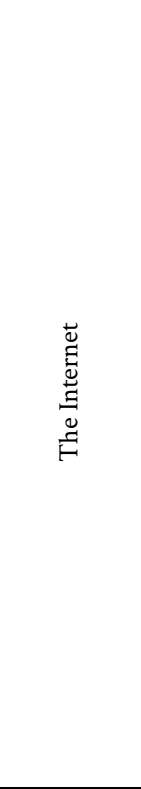 } & Limitless, endless & $\begin{array}{l}\text { library, ocean, universe, infinity, earth, sea, octopus, encyclopedia, humans, spider, toolbox, grocer, knowledge } \\
\text { accumulation, wise man, room full of information, city, cloud, abyss, elephant, life, light speed, book, bookshelf, } \\
\text { greengrocer's, teacher, pyramid, magician, magic box, magic broom, historic item, land, space, Josph Morgan, } \\
\text { Mustafa Topaloğlu, Spiderman }\end{array}$ \\
\hline & Making life easy & $\begin{array}{l}\text { book, library, Alaaddin's magic lamp, Superman, phone, encyclopedia, animal, } 911 \text { service, mother, father, car, } \\
\text { friend, wise man, Gargamel, newspaper, sister Güzin, wise engine, St. Hizir, servant, rooms, dictionary, } \\
\text { communication network, speaking, Nasreddin Hodja, Spiderman, Robin Hood, plane, vehicles }\end{array}$ \\
\hline & Necessities & $\begin{array}{l}\text { water, food, life, breathing, salt, hunger, couch, most-liked item, chocolate, house, stuffed mutton balls, ladle, } \\
\text { mother, funfair }\end{array}$ \\
\hline & Both beneficial and harmful & $\begin{array}{l}\text { elephant and rabbit, horse, knife, bear, monster, Pandora's box, circle, vacuum cleaner, high heel shoe, virus, meal, } \\
\text { beneficial substance in alcohol }\end{array}$ \\
\hline & Organs & brain, veins, nerve cells \\
\hline & Addictive items & car, makeup, cigarette, air and water, drugs \\
\hline & Harmful items & illness, monster, virus, dump site \\
\hline & Slow animals & turtle \\
\hline & Fast animals & horse, ant \\
\hline & Developing and changing & Che Guevera, Historic identity \\
\hline & Means of communication & bird, carrier pigeon \\
\hline & Beneficial items & fun club \\
\hline & Food & chocolate \\
\hline
\end{tabular}


Table 2. Categories formed for the keywords (continued)

\begin{tabular}{|c|c|c|}
\hline Keywords & Conceptual Categories & Metaphor Names \\
\hline \multirow{12}{*}{ 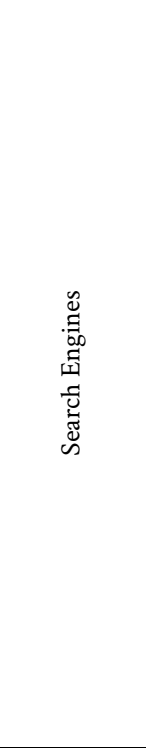 } & Making life easy & $\begin{array}{l}\text { library, encyclopedia, teacher, Superman, vehicle, assistant, book, magnifying glass , Esra Erol, servant, woman, dog, } \\
\text { girl, hero, family, smart devices, key, mother, father, parents, mirror, genius mind, easiness, electronic goods, factory } \\
\text { workers, map genie, human mind, jet, introduction part, greengrocer, price tag, tongs, angel, stairs, fruit pieces, } \\
\text { Nasreddin Hodja's donkey, cooker, compass, clock, exhibition, Sherlock Holmes, dictionary, pilot, flying carpet, old } \\
\text { wise man }\end{array}$ \\
\hline & Limitless, endless & $\begin{array}{l}\text { wise man, library, encyclopedia, woman, earth, archive, shopping center, sea, lady's bag , herbalist, researcher, } \\
\text { bucket, wise sister, boutique, dustbin, mountains, matchmaking program, Saint Çelebi, Saint, philosopher, lake, life, } \\
\text { book, Müge Anl, Nasreddin Hodja, school, forest, Spiderman's web, Pepe, Father Smurf, chips with taso, space, old } \\
\text { man }\end{array}$ \\
\hline & Beneficial items & hero of a fairy tale, Tom and Jerry, friend, teacher, milkman, cow, light, antibiotics, bridge, information \\
\hline & Hardworking animals & ant, bee \\
\hline & Harmful items & spoilt child, alcohol seller \\
\hline & Both beneficial and harmful & spy, friend \\
\hline & Vehicles & ship, vehicle \\
\hline & Addictive items & alcoholic drink \\
\hline & Fast animals & cheetah \\
\hline & Small animals & bird \\
\hline & Tools & jigsaw puzzle \\
\hline & Necessities & tree \\
\hline \multirow{8}{*}{ 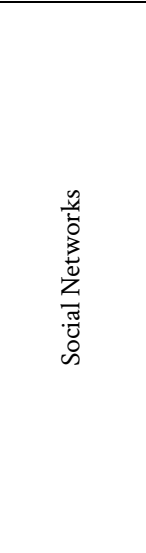 } & Means of Communication & $\begin{array}{l}\text { letter, café, pigeon, telephone, gossip, friends' gathering, easter, gold day, friends circle, nightingale, rail line, } \\
\text { wedding, diary, communication, communication skill, communication device, wire, identity, book, bird, school, } \\
\text { holiday resort, party, window, public transport vehicle, train station }\end{array}$ \\
\hline & Addictive items & $\begin{array}{l}\text { octopus , spider web, cigarette, drug, prison, swamp, alcohol, lion, independence, Burak Özçivit, ripped stocking, } \\
\text { whirlpool, pit, creature, sedative }\end{array}$ \\
\hline & Necessities & $\begin{array}{l}\text { family, neighbours, friend, alcohol addict, mother, friend circle, gossip, man, matchmaking program, heart, bird, } \\
\text { model, happiness, favourite toy, water }\end{array}$ \\
\hline & Making life easy & $\begin{array}{l}\text { octopus, humans, newspaper boy, journalist, fish, meeting point, café, flat, pigeon, extended family, small child, hero, } \\
\text { spider, popular friend, fan group }\end{array}$ \\
\hline & Beneficial items & toy, café, game, leisure center, diary, air, spider web, funfair, university \\
\hline & Harmful items & $\begin{array}{l}\text { parrot, notebook of preschool children, big animal, monster, gossipy women, turtle, koala, labyrinth, cigarette, } \\
\text { endless road, chicken hen }\end{array}$ \\
\hline & Limitless, endless & octopus, spider web, funfair, lace, zoo \\
\hline & Both beneficial and harmful & candy, dishonest person, herd, shopping mall, slippery bridge \\
\hline \multirow{13}{*}{ 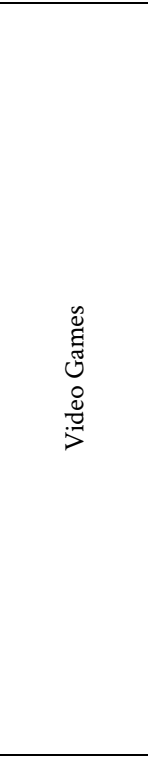 } & Developing and changing & postman, virus \\
\hline & Food & dinner, apple \\
\hline & Organs & vein \\
\hline & Small animals & spider \\
\hline & Addictive items & $\begin{array}{l}\text { drugs, cigarette, chocolate, alcohol, heroine, coffee, lion, darling , fire, addiction, addictive substances, myself, illness, } \\
\text { beer, chocolate, crossword, Burak Özçivit, monster, chips, seed, playing house, well, book, cocaine, pasta, nicotine, } \\
\text { chewing gum, sugar, collar, Teen Wolf, television, meal }\end{array}$ \\
\hline & Beneficial items & $\begin{array}{l}\text { funfair, friends, toy, dreams, life, game boy, playing on the street, an item, Alice in Wonderland, myself, Bugs Bunny, } \\
\text { lifeguard, Çallkuşu, chocolate, playground, game, leisure center, Fenerbahçe, football, excitement, coffee, hero, book } \\
\text { of tales, listening to music, game room, game, deadly illness, psychologist, clock }\end{array}$ \\
\hline & Harmful items & $\begin{array}{l}\text { sleep, monster, television, knitting, appendix, empty plate, useless dream, empty room, Fenerbahçe, faux pas, } \\
\text { unnecessary task, working in vain, dream world, thief, empty flat, cola, puppet, sly friend, dry tissue, painting nails, } \\
\text { cigarette, indispensability, tuberculosis, snake, devices stealing time, passing time, time machine, poison }\end{array}$ \\
\hline & Both beneficial and harmful & dream, bacteria, chocolate, cat, funfair, appetizer, useless item, clock, television, passion, dungeon \\
\hline & Limitless, endless & funfair, fishnet, earth, leisure center, gloves, animals, small organisms \\
\hline & Developing and changing & Tom and Jerry, virus, graveyard \\
\hline & Fast animals & squirrel \\
\hline & Making life easy & friend \\
\hline & Necessities & meal \\
\hline
\end{tabular}


Table 3. Frequencies and Percentages of Categories formed for the keywords

Conceptual Categories

Developing and changing

Limitless, endless

Making life easy

Necessities

Beneficial items

Tools

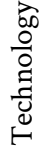

Both beneficial and harmful

Rapidly progressing

Small animals

Food

Harmful items

Vehicles

Hardworking animals

Big animals

Addictive items

Total:

Making life easy

Organs

Limitless, endless

Tools

Necessities

Beneficial items

Both beneficial and harmful

氙 Fast animals

Harmful items

ن Addictive items

Big animals

Developing and changing

Vehicles

Hardworking animals

Means of communication

Small animals

Total:

Limitless, endless

Making life easy

Necessities

Both beneficial and harmful

Organs

$\stackrel{\Xi}{\Xi} \quad$ Addictive items

Harmful items

Slow animals

Fast animals

Developing and changing

Means of communication

Beneficial items

Food

Total:
Metaphor Frequency

Metaphor Percentage

$\begin{array}{ll}56 & 36,3 \\ 18 & 11,6\end{array}$

15

14

11

9

7

7

5

5

3

3

3

2

2

1

1

154

36

29

19

14

13

11

5

5

4

4

3

3

3

3

3

2

1

1

153

69

38

25

13

8

5

5

4

3

2

2

2

1

1

173

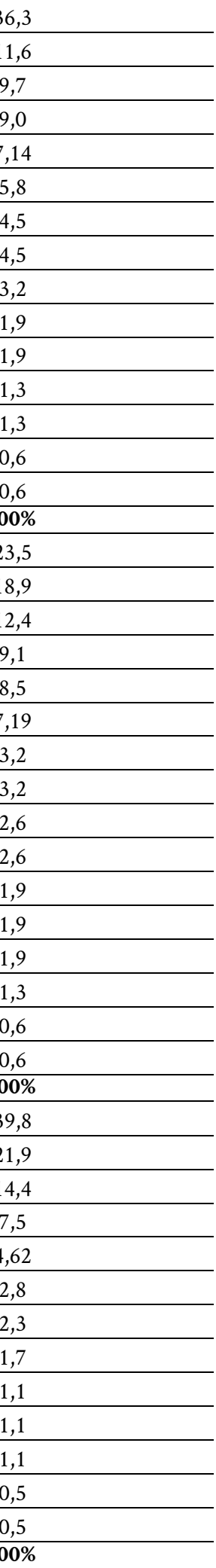


Table 3. Frequencies and Percentages of Categories formed for the keywords (continued)

\begin{tabular}{|c|c|c|c|}
\hline \multirow{13}{*}{ 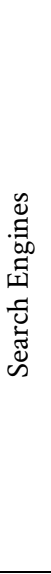 } & Making life easy & 86 & 50,8 \\
\hline & Limitless, endless & 57 & 33,7 \\
\hline & Beneficial items & 10 & 5,9 \\
\hline & Hardworking animals & 5 & 2,9 \\
\hline & Harmful items & 2 & 1,1 \\
\hline & Both beneficial and harmful & 2 & 1,1 \\
\hline & Vehicles & 2 & 1,1 \\
\hline & Addictive items & 1 & 0,5 \\
\hline & Fast animals & 1 & 0,5 \\
\hline & Small animals & 1 & 0,5 \\
\hline & Tools & 1 & 0,5 \\
\hline & Necessities & 1 & 0,5 \\
\hline & Total: & 169 & $100 \%$ \\
\hline \multirow{13}{*}{ 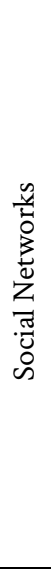 } & Means of Communication & 54 & 33,3 \\
\hline & Addictive items & 21 & 12,9 \\
\hline & Necessities & 19 & 11,7 \\
\hline & Making life easy & 18 & 11,1 \\
\hline & Beneficial items & 15 & 9,2 \\
\hline & Harmful items & 12 & 7,4 \\
\hline & Limitless, endless & 10 & 6,1 \\
\hline & Both beneficial and harmful & 5 & 3,0 \\
\hline & Developing and changing & 2 & 1,2 \\
\hline & Food & 2 & 1,2 \\
\hline & Organs & 2 & 1,2 \\
\hline & Small animals & 2 & 1,2 \\
\hline & Total: & 162 & $100 \%$ \\
\hline \multirow{10}{*}{ 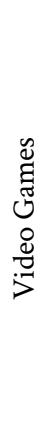 } & Addictive items & 62 & 37,3 \\
\hline & Beneficial items & 44 & 26,5 \\
\hline & Harmful items & 33 & 19,8 \\
\hline & Both beneficial and harmful & 12 & 7,2 \\
\hline & Limitless, endless & 9 & 5,4 \\
\hline & Developing and changing & 3 & 1,8 \\
\hline & $\underline{\text { Fast animals }}$ & 1 & 0,6 \\
\hline & Making life easy & 1 & 0,6 \\
\hline & Necessities & 1 & 0,6 \\
\hline & Total: & 166 & $100 \%$ \\
\hline
\end{tabular}


Table 4. Participants' Justifications for the most common metaphors

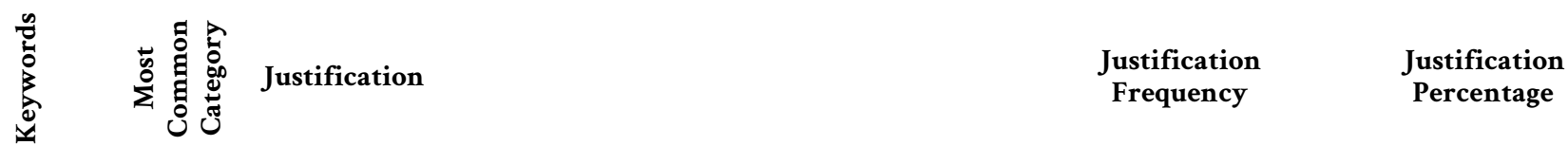

\begin{tabular}{|c|c|c|c|c|}
\hline \multirow{5}{*}{ 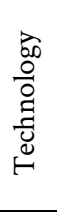 } & \multirow{5}{*}{ 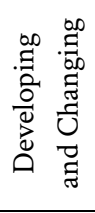 } & because it evolves continuously & 38 & 67,8 \\
\hline & & because it changes continuously & 12 & 21,4 \\
\hline & & because it renews itself & 4 & 7,1 \\
\hline & & because it develops itself & 2 & 3,5 \\
\hline & & Total: & 56 & $100 \%$ \\
\hline \multirow{11}{*}{ 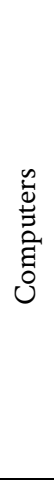 } & \multirow{11}{*}{ 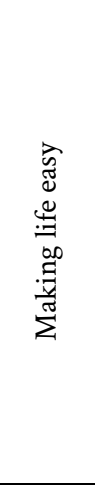 } & because they store information efficiently & 10 & 27,7 \\
\hline & & because they help us & 9 & 25,0 \\
\hline & & because they are multifunctional & 9 & 25,0 \\
\hline & & because they do everything fast & 2 & 5,5 \\
\hline & & because they shape you & 1 & 2,7 \\
\hline & & because they solve problems easily & 1 & 2,7 \\
\hline & & because they answer questions & 1 & 2,7 \\
\hline & & because they are more superior than humans & 1 & 2,7 \\
\hline & & because they keep everything in memory & 1 & 2,7 \\
\hline & & because they teach us & 1 & 2,7 \\
\hline & & Total: & 36 & $100 \%$ \\
\hline \multirow{13}{*}{ 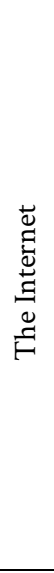 } & \multirow{13}{*}{ 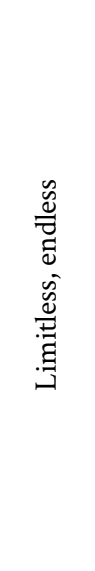 } & because it has all we need & 27 & 39,1 \\
\hline & & because it contains lots of information & 18 & 26,0 \\
\hline & & because it has no restriction or boundary & 8 & 11,5 \\
\hline & & because it is quick and comprehensive & 4 & 5,8 \\
\hline & & because it is like a web & 3 & 4,3 \\
\hline & & because it is very large & 3 & 4,3 \\
\hline & & because it helps our learning process & 1 & 1,4 \\
\hline & & because it answers questions & 1 & 1,4 \\
\hline & & because it is everywhere & 1 & 1,4 \\
\hline & & because it looks endless but actually it is not & 1 & 1,4 \\
\hline & & because it is like a city & 1 & 1,4 \\
\hline & & because it is mysterious & 1 & 1,4 \\
\hline & & Total: & 69 & $100 \%$ \\
\hline \multirow{18}{*}{ 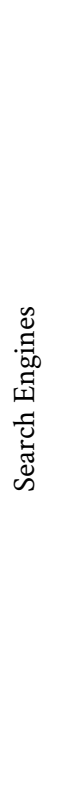 } & \multirow{18}{*}{ 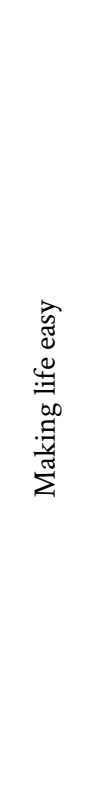 } & because we can find all we are looking for & 29 & 33,7 \\
\hline & & because they help us & 12 & 13,9 \\
\hline & & because they answer our questions & 8 & 9,3 \\
\hline & & because they meet our needs & 8 & 9,3 \\
\hline & & because they know everything & 5 & 5,8 \\
\hline & & because they provide access to knowledge & 5 & 5,8 \\
\hline & & because they take you to everywhere & 4 & 4,6 \\
\hline & & because they lead to the target & 3 & 3,4 \\
\hline & & because they offer choices & 2 & 2,3 \\
\hline & & because they make access to knowledge easier & 2 & 2,3 \\
\hline & & because they enhance learning & 2 & 2,3 \\
\hline & & because they select useful knowledge & 1 & 1,1 \\
\hline & & because they maintain communication & 1 & 1,1 \\
\hline & & because they are smart & 1 & 1,1 \\
\hline & & because they provide results if you use them well & 1 & 1,1 \\
\hline & & because they are different from human mind & 1 & 1,1 \\
\hline & & because they are companions & 1 & 1,1 \\
\hline & & Total: & 86 & $100 \%$ \\
\hline
\end{tabular}


Table 4. Participants' Justifications for the most common metaphors (continued)

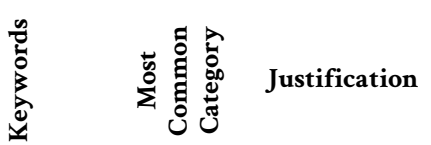

Justification Frequency Justification Percentage

\begin{tabular}{|c|c|c|c|c|}
\hline \multirow{11}{*}{ 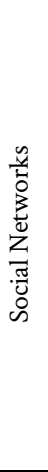 } & \multirow{11}{*}{ 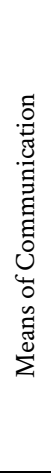 } & because they put us in communication with other people & 25 & 46,3 \\
\hline & & because they make communication easier & 5 & 9,2 \\
\hline & & because they help communication & 5 & 9,2 \\
\hline & & because they enable us to share things with people & 5 & 9,2 \\
\hline & & because they enable us to meet new people & 4 & 7,4 \\
\hline & & because they bring friends living far away closer & 2 & 3,7 \\
\hline & & because they provide us with personal information & 2 & 3,7 \\
\hline & & because they provide opportunities to exchange information & 2 & 3,7 \\
\hline & & because they provide a new environment & 1 & 1,8 \\
\hline & & because they enhance curiousity & 1 & 1,8 \\
\hline & & Total: & 54 & $100 \%$ \\
\hline \multirow{10}{*}{ 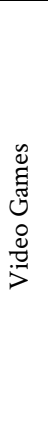 } & \multirow{10}{*}{ 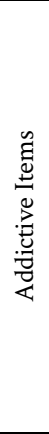 } & because they cause addiction & 34 & 54,8 \\
\hline & & because they occupy you deeply & 12 & 19,3 \\
\hline & & because you want to eat more & 4 & 6,4 \\
\hline & & because they cause loss of time and money & 3 & 4,8 \\
\hline & & because you can't give up although they are harmful & 2 & 3,2 \\
\hline & & because they make you happy & 1 & 1,6 \\
\hline & & because they are like an epidemic & 1 & 1,6 \\
\hline & & because they never fully satisfy you & 1 & 1,6 \\
\hline & & because they are harmful & 1 & 1,6 \\
\hline & & Total: & 62 & $100 \%$ \\
\hline
\end{tabular}

\section{REFERENCES}

Gecer, A. (2013). Determination of the computer self-efficacy perception of students and metaphors related to "computer ownership" TOJET: The Turkish Online Journal of Educational Technology, 12(3), 51-71.

Gurol, M., \& Donmus, V. (2010). Metaphors created by prospective teachers related to the concept of "Social Network". Procedia Social and Behavioural Sciences, 9(2010), 1489-1496. https://doi.org/10.1016/j.sbspro.2010.12.354

Jansen, H. (2010). The Logic of Qualitative Survey Research and its Position in the Field of Social Research Methods. Forum Qualitative Sozialforschung / Forum: Qualitative Social Research, 11(2). Art. 11. https://doi.org/10.17169/fqs-11.2.1450
Kobak, M., \& Taskin, N. R. (2012). Prospective Teachers' Perceptions of Technology in Three Diffferent Ways. Procedia Social and Behavioural Sciences, 46(2012), 3629-3636. https://doi.org/10.1016/ j.sbspro.2012.06.118

Koc, M. (2013). Student teachers' concepts of technology: A metaphor analysis. Computers \& Education, 68(2013), 1-8. https://doi.org/ 10.1016/j.compedu.2013.04.024

Morrison, W. F., \& Jeffs, T. L. (2005). Outcomes of preservice teacher's technology use. Assistive Technology Outcomes and Benefits, 2(1), 7178.

Saban, A., Kocbeker, B. N., \& Saban, A. (2006). Prospective Teachers' Conceptions of Teaching and Learning Revealed Through Metaphor Analysis. Learning and Instruction, 17(2006), 123e139. https://doi.org/10.1016/j.learninstruc.2007.01.003

Teo, T., \& Lee, C. B. (2010). Explaining the intention to use technology among student teachers: An application of the theory of planned behavior. Campus-Wide Information Systems, 27(2), 60-67. https://doi.org/10.1108/10650741011033035 